\title{
Visibility of lattice points
}

\author{
by \\ Yong-Gao Chen (Nanjing) and Lin-Feng Cheng (Xuzhou)
}

1. Introduction. Two integer points $P\left(a_{1}, \ldots, a_{k}\right)$ and $Q\left(b_{1}, \ldots, b_{k}\right)$ are said to be visible to each other if either $P=Q$ or there are no other integer points on the line segment joining $P$ and $Q$. It is not difficult to verify that if $P \neq Q$, then $P$ and $Q$ are visible to each other if and only if $\operatorname{gcd}\left(a_{1}-b_{1}, \ldots, a_{k}-b_{k}\right)=1$. We say that an integer point set $A$ is visible from an integer point set $B$ if each point of $A$ is visible from some point of $B$.

For $k \geq 2$, let

$$
\Delta_{n}^{k}=\left\{\left(x_{1}, \ldots, x_{k}\right): x_{i} \text { integers and } 1 \leq x_{i} \leq n(1 \leq i \leq n)\right\} .
$$

Define

$$
\begin{aligned}
f_{k}(n) & =\min \left\{|S|: S \subset \mathbb{Z}^{k}, \Delta_{n}^{k} \text { is visible from } S\right\}, \\
F_{k}(n) & =\min \left\{|S|: S \subseteq \Delta_{n}^{k}, \Delta_{n}^{k} \text { is visible from } S\right\} .
\end{aligned}
$$

It is clear that $f_{k}(n) \leq F_{k}(n)$. Erdős, Gruber and Hammer [4] asked for an explicit construction of $S$ such that $S \subset \Delta_{n}^{2},|S|=O(\log n)$ and $\Delta_{n}^{2}$ is visible from $S$. A better construction of $S$ was given by Adhikari and Balasubramanian [2]. We have

$$
\begin{array}{lr}
F_{2}(n) \geq \frac{1}{2} \frac{\log n}{\log \log n}, \quad n \geq n_{0}, & \text { (Abbott [1]) } \\
F_{2}(n)=O\left(\frac{\log n \log \log \log n}{\log \log n}\right), & \text { (Adhikari, Balasubramanian [2]) } \\
F_{k}(n)=O\left(\frac{\log n}{\log \log n}\right), \quad k \geq 3, & \text { (Adhikari, Chen [3]) }
\end{array}
$$

2000 Mathematics Subject Classification: 11H99, 11B75.

Supported by the National Natural Science Foundation of China, Grant No. 10171046 and the Teaching and Research Award Program for Outstanding Young Teachers in Nanjing Normal University. 
In fact, the method in Abbott [1] implies that

$$
F_{k}(n) \geq f_{k}(n) \geq \frac{1}{2} \frac{\log n}{\log \log n}, \quad n \geq n_{0}, \quad \text { for all } k \geq 2 .
$$

Thus, for $k \geq 3$, the main orders of $f_{k}(n)$ and $F_{k}(n)$ are $\log n / \log \log n$. In this note we are interested in the constant factors. Let

$$
\zeta(k)=\sum_{m=1}^{\infty} \frac{1}{m^{k}}=\prod_{p}\left(1-\frac{1}{p^{k}}\right) .
$$

The following results are proved.

TheOrem 1. For $k \geq 2$ we have

$$
f_{k}(n) \geq \zeta(k) \frac{\log n}{\log \log n}(1+o(1))
$$

TheOREM 2. For $k \geq 3$ we have

$$
F_{k}(n) \leq \zeta(k-1) \frac{\log n}{\log \log n}(1+o(1)) .
$$

REMARK. The first author conjectures that

$$
f_{k}(n)=\zeta(k) \frac{\log n}{\log \log n}(1+o(1)), \quad F_{k}(n)=\zeta(k) \frac{\log n}{\log \log n}(1+o(1)) .
$$

By an analogous argument to the proof of Theorem 2, we can prove that the conjecture for $k \geq 3$ follows from the conjecture that for every fixed $s$,

$$
\max _{s<m \leq n} \omega((m-1)(m-2) \ldots(m-s))=(1+o(1)) \frac{\log n}{\log \log n},
$$

and the conjecture for $k=2$ follows from the conjecture that

$$
\max _{s_{n}<m \leq n} \omega\left((m-1)(m-2) \ldots\left(m-s_{n}\right)\right)=(1+o(1)) \frac{\log n}{\log \log n},
$$

where $s_{n}=[2 \log n / \log \log n]$.

2. Proofs. Let $p_{1}, p_{2}, \ldots$ be all positive primes in increasing order, that is, $p_{1}=2, p_{2}=3, \ldots$ As usual, we will use $p$ to denote a prime. For two points $P$ and $Q$ in $\mathbb{Z}^{k}$ and an integer $m$, we say that $P$ and $Q$ are congruent $\bmod m$ if all coordinates are congruent $\bmod m$.

Lemma 1. Let $B$ be a finite subset of $\mathbb{Z}^{k}$. Then there exist at least $|B| / p^{k}$ points in $B$ which are congruent mod $p$.

Proof. Lemma 1 follows from the fact that points in $\mathbb{Z}^{k} \bmod p$ has $p^{k}$ different possible values. 
Proof of Theorem 1. Let $0<\varepsilon<1 / 8$. We take an integer $t$ such that

$$
\prod_{i=1}^{t}\left(1-\frac{1}{p_{i}^{k}}\right) \leq \zeta(k)^{-1}(1+\varepsilon) .
$$

For $n \geq n_{1}(\varepsilon, k)$ there exists an integer $r$ such that

$$
\zeta(k) \frac{\log n}{\log \log n}(1-6 \varepsilon) \leq r \leq \zeta(k) \frac{\log n}{\log \log n}(1-5 \varepsilon), \quad p_{1}^{k} p_{2}^{k} \ldots p_{t}^{k} \mid r .
$$

Suppose that $Q_{1}, \ldots, Q_{r}$ are $r$ distinct points in $\mathbb{Z}^{k}$. By Lemma 1 there exists $A_{1} \subseteq\left\{Q_{1}, \ldots, Q_{r}\right\}$ such that $\left|A_{1}\right|=r / p_{1}^{k}$ and the points in $A_{1}$ are congruent $\bmod p_{1}$. Let $B_{1}=\left\{Q_{1}, \ldots, Q_{r}\right\} \backslash A_{1}$. Then $\left|B_{1}\right|=\left(1-1 / p_{1}^{k}\right) r$ is divisible by $p_{2}^{k}$. By Lemma 1 there exists $A_{2} \subseteq B_{1}$ such that $\left|A_{2}\right|=\left|B_{1}\right| / p_{2}^{k}$ and the points in $A_{2}$ are congruent $\bmod p_{2}$. Let $B_{2}=B_{1} \backslash A_{2}$. Then $\left|B_{2}\right|=$ $\left(1-1 / p_{2}^{k}\right)\left(1-1 / p_{1}^{k}\right) r$ is divisible by $p_{3}^{k}$. Similarly, we obtain $A_{3}, \ldots, A_{t}$ and $B_{3}, \ldots, B_{t}$ such that $\left|A_{i}\right|=\left|B_{i-1}\right| / p_{i}^{k}, B_{i}=B_{i-1} \backslash A_{i}$ and the points in $A_{i}$ are congruent $\bmod p_{i}$ for $3 \leq i \leq t$. Then

$$
\left|B_{t}\right|=\left(1-\frac{1}{p_{t}^{k}}\right)\left|B_{t-1}\right|=\ldots=r \prod_{i=1}^{t}\left(1-\frac{1}{p_{i}^{k}}\right) \leq \frac{\log n}{\log \log n}(1-4 \varepsilon) .
$$

Hence, for $n \geq n_{2}(\varepsilon, k)$,

$$
t+\left|B_{t}\right| \leq \frac{\log n}{\log \log n}(1-3 \varepsilon) .
$$

Let $s=t+\left|B_{t}\right|$. Let $B_{t}=A_{t+1} \cup \ldots \cup A_{s}$ with $\left|A_{i}\right|=1$ and $A_{i} \cap A_{j}=\emptyset$ for $t+1 \leq i, j \leq s$ and $i \neq j$. By the Chinese Remainder Theorem, there exists a point $Q$ in $\Delta_{(r+1) p_{1} \ldots p_{s}}^{k}$ which is different from $Q_{1}, \ldots, Q_{r}$ and congruent to the points of $A_{i} \bmod p_{i}$ for each $i$. For $n \geq n_{3}(\varepsilon, k)$ by (1) we have

$$
\begin{aligned}
\log \left((r+1) p_{1} \ldots p_{s}\right) & \leq \log (r+1)+s \log p_{s} \\
& \leq \varepsilon \log n+(1-2 \varepsilon) \log n<\log n .
\end{aligned}
$$

Hence, $Q \in \Delta_{n}^{k}$ and $Q$ is invisible from any point of $Q_{1}, \ldots, Q_{r}$. Therefore

$$
f_{k}(n)>r \geq \zeta(k) \frac{\log n}{\log \log n}(1-6 \varepsilon) .
$$

This completes the proof of Theorem 1 .

Proof of Theorem 2. Let $0<\varepsilon<1 / 4$. Let $t$ be an integer with

$$
2^{k-1} \sum_{p>p_{t}} \frac{1}{p^{k-1}}<\varepsilon \zeta(k-1)^{-1}
$$

For $n \geq n_{4}(\varepsilon, k)$ there exists an integer $r$ with

$$
\zeta(k-1) \frac{\log n}{\log \log n} \frac{1+2 \varepsilon}{1-\varepsilon} \leq r^{k-1} \leq \zeta(k-1) \frac{\log n}{\log \log n} \frac{1+3 \varepsilon}{1-\varepsilon}, \quad p_{1} p_{2} \ldots p_{t} \mid r .
$$


Let

$$
\begin{aligned}
G_{n}= & \left\{\left(a_{1}, \ldots, a_{k-1}, 1\right): a_{i} \text { integers, } 1 \leq a_{i} \leq r(1 \leq i \leq k-1)\right\} \\
& \cup\{(2,2, \ldots, 2)\} .
\end{aligned}
$$

Given any point $\left(x_{1}, \ldots, x_{k}\right) \in \Delta_{n}^{k}$. If $x_{k}=1$, then $\left(x_{1}, \ldots, x_{k}\right)$ is visible from $(2,2, \ldots, 2)$. Now we assume that $x_{k}>1$. We will show that there exists at least one point $\left(a_{1}, \ldots, a_{k-1}, 1\right) \in G_{n}$ such that

$$
\left(x_{1}-a_{1}, \ldots, x_{k-1}-a_{k-1}, x_{k}-1\right)=1 .
$$

In order to prove this, we use a simple sieving argument. Let $q_{1}, \ldots, q_{m}$ be the prime divisors of $x_{k}-1$. We know that

$$
m=\omega\left(x_{k}-1\right) \leq(1+o(1)) \log n / \log \log n .
$$

We want to find $\left(a_{1}, \ldots, a_{k-1}, 1\right) \in G_{n}$ so that no $q_{i}$ divides each $x_{j}-a_{j}, 1 \leq$ $j \leq k-1$.

For the primes $q_{j}$ that are among $p_{1}, \ldots, p_{t}$ we use the combinatorial sieve. We find that the number of remaining vectors is

$$
r^{k-1} \prod\left(1-p_{i}^{-(k-1)}\right)>\zeta(k-1)^{-1} r^{k-1} .
$$

Each prime $q_{j}$ with $p_{t}<q_{j} \leq r$ excludes at most $\left(1+\left[r / q_{j}\right]\right)^{k-1}<\left(2 r / q_{j}\right)^{k-1}$ vectors. The total number of these is

$$
<(2 r)^{k-1} \sum_{q_{j}>p_{t}} q_{j}^{-(k-1)}<\varepsilon \zeta(k-1)^{-1} r^{k-1} .
$$

Finally, a $q_{j}>r$ excludes at most one, altogether $(1+o(1)) \log n / \log \log n$ at most. Since

$$
\zeta(k-1)^{-1} r^{k-1}>(1+\varepsilon) \frac{\log n}{\log \log n}+\varepsilon \zeta(k-1)^{-1} r^{k-1},
$$

we are done.

Therefore

$$
F_{k}(n) \leq\left|G_{n}\right|+1 \leq r^{k-1}+1 \leq \zeta(k-1) \frac{1+3 \varepsilon}{1-\varepsilon} \frac{\log n}{\log \log n}+1 .
$$

This completes the proof.

Acknowledgements. I am grateful to the referee for his/her suggestions to shorten the proof of Theorem 2 and to add more remarks pertaining to Theorem 2.

\section{References}

[1] H. L. Abbott, Some results in Combinatorial Geometry, Discrete Math. 9 (1974), 199-204. 
[2] S. D. Adhikari and R. Balasubramanian, On a question regarding visibility of lattice points, Mathematika 43 (1996), 155-158.

[3] S. D. Adhikari and Y.-G. Chen, On a question regarding visibility of lattice points, II, Acta Arith. 89 (1999), 279-282.

[4] P. Erdős, P. M. Gruber and J. Hammer, Lattice Points, Pitman Monographs Surveys Pure Appl. Math. 39, Wiley, New York, 1989.

Department of Mathematics

Nanjing Normal University

Nanjing 210097, China

E-mail: ygchen@pine.njnu.edu.cn
Department of Mathematics China University of Mining and Technology Xuzhou 221008, China E-mail: cumtclf@sina.com.cn

Received on 14.11.2001

and in revised form on 26.8.2002 